
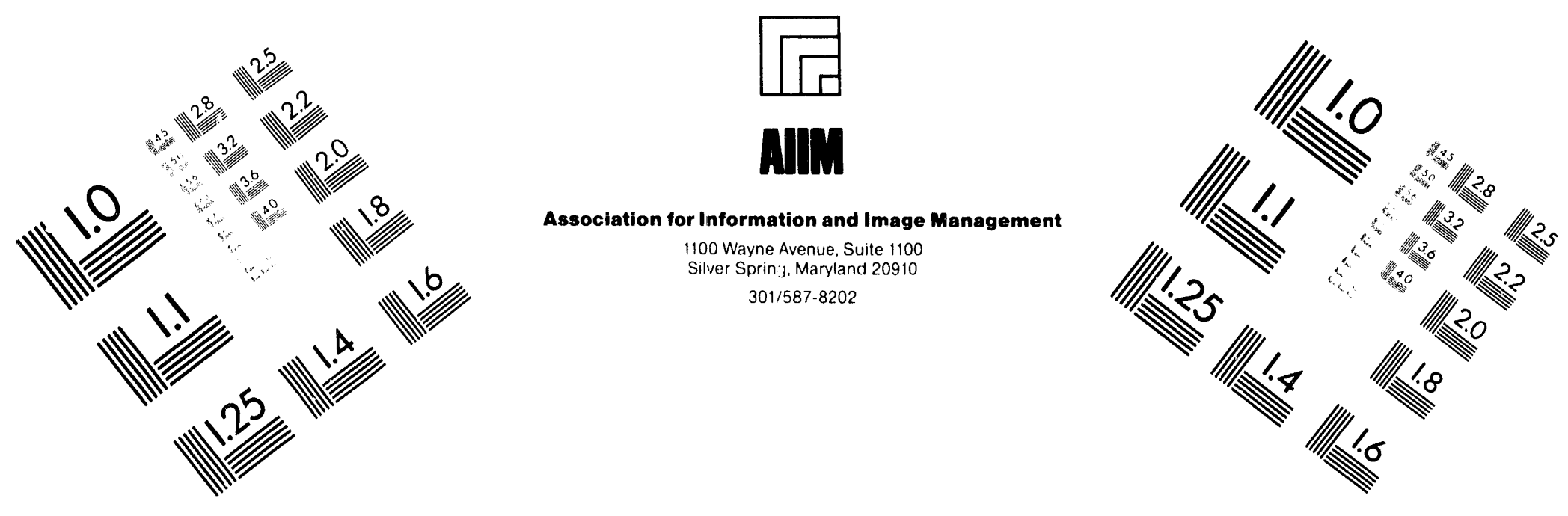

\title{
Centimeter
}

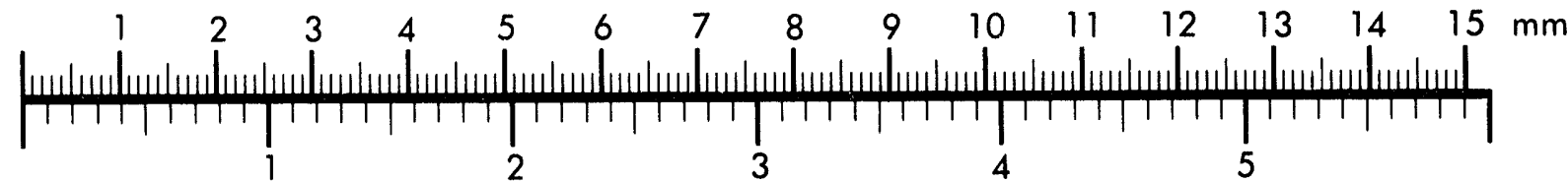

Inches
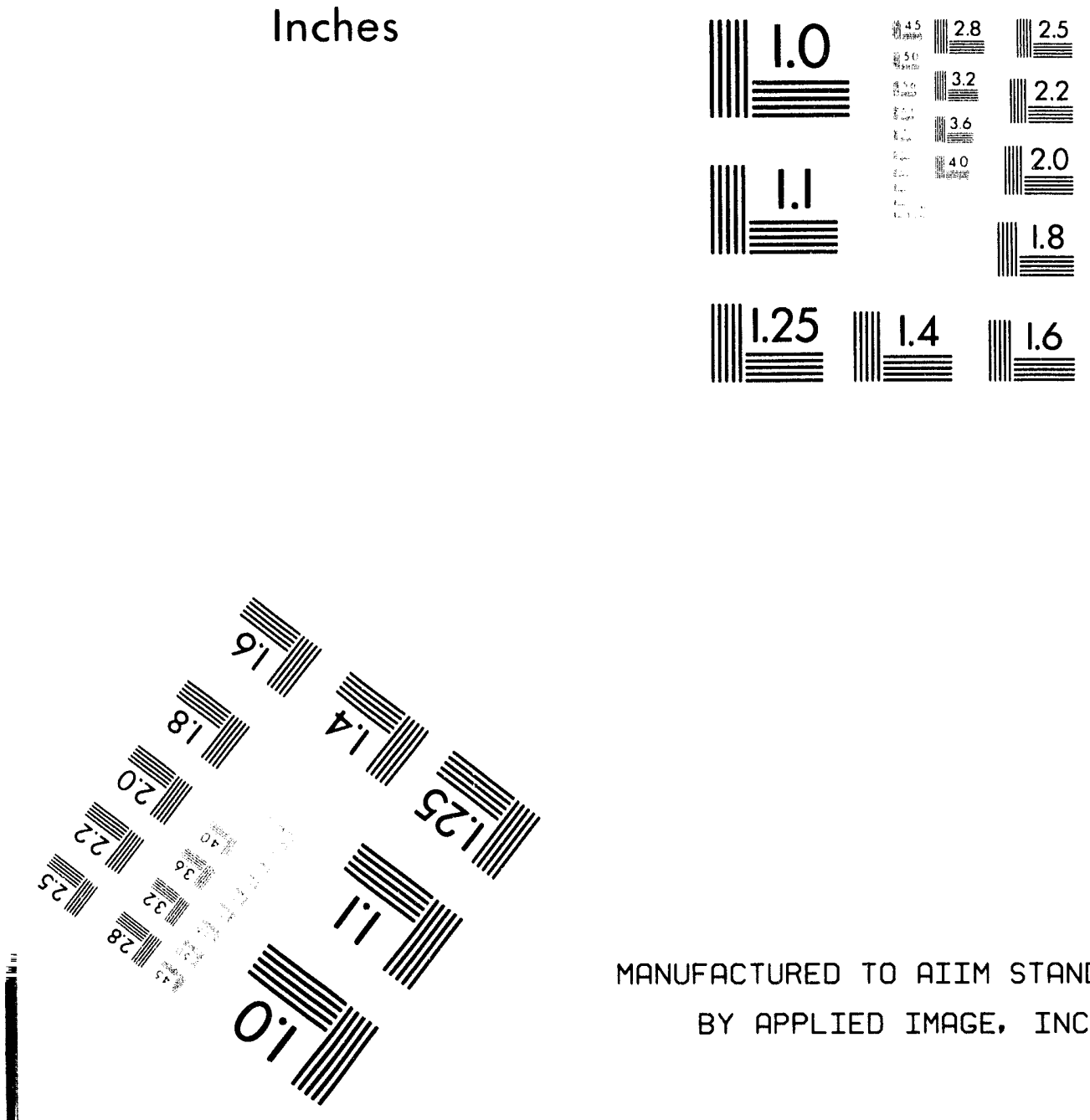

MANUFACTURED TO AIIM STANDARDS

BY APPLIED IMAGE, INC.

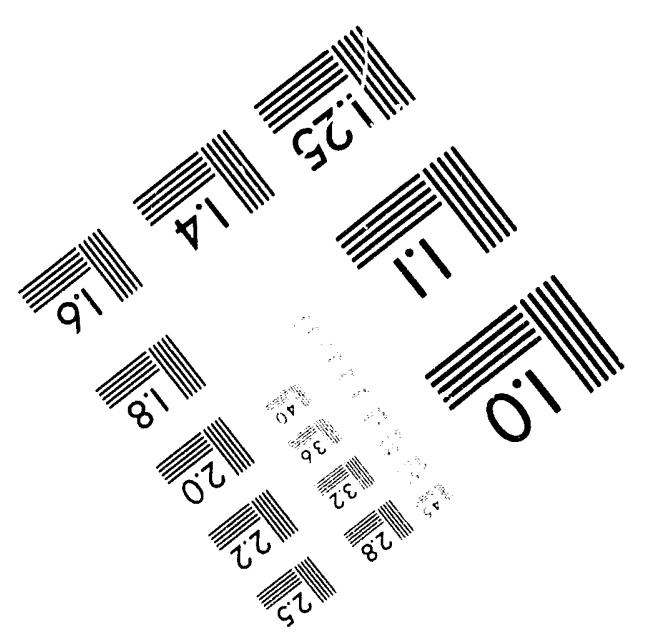


WSRC-RP-94-250

Key Words Dose

AXAIR89Q

SAR

JCO

Retention: Lifetime

\section{UPDATE OF HB-LINE CONSEQUENCES BASED ON NEW METEOROLOGICAL AND POPULATION DATABASES IN $\operatorname{AXAIR89Q}(\mathbf{U})$}

M.E. DelGenio

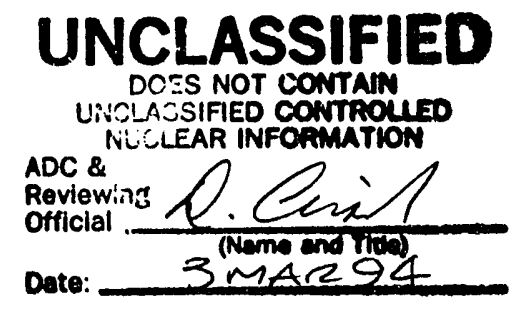

Issued: March 1994

SRTC

SAVANNAH RIVER TECHNOLOGY CENTER

Westinghouse Savannah River Company

Savannah River Site

Aiken, SC 29808

PREPARED FOR THE U.S. DEPARTME] IT OF ENERGY UNDER CONTACT NO. DE-AC09-89SR 18035

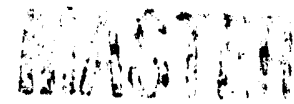




\section{DISCLAIMER}

This report was prepared as an account of work sponsored by an agency of the United States Government. Neither the United States Government nor any agency thereof, nor any of their employees, makes any warranty, express or implied, or assumes any legal liability or responsibility for the accuracy, completeness, or usefulness of any information, apparatus, product, or process disclosed, or represents that its use would not infringe privately owned rights. Reference herein to any specific commercial product, process, or service by trade name, trademark, manufacturer, or otherwise does not necessarily constitute or imply its endorsement, recommendation, or favoring by the United States Government or any agency thereof. The views and opinions of authors expressed herein do not necessarily state or reflect those of the United. States Government or any agency thereof.

This report has been reproduced directly from the best available copy.

Available to DOE and DOE contractors from the Office of Scientific and Technical Information, P. O. Box 62, Oak Ridge, TN 37831; prices available from (615) 576-8401.

Available to the public from the National Technical Information Service, U. S. Department of Commerce, 5285 Port Royal Rd., Springfield, VA 22161 
Document: $\quad$ WSRC-RP-94-250

Title:

Update Of HB-Line Consequences Based on New Meteorological and Population Databases in AXAIR89Q (U)

Approvals:

$\frac{M \cdot G \cdot \text { Delsy }}{\text { M.E. DelGenio, Author }}$

X. R. o.the

K.R. O'Kula

Manager, Data and Consequence Analysis

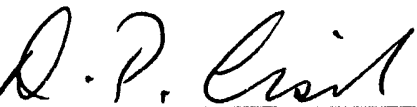

D.P. Eisele

Acting Manager, Separations Analysis

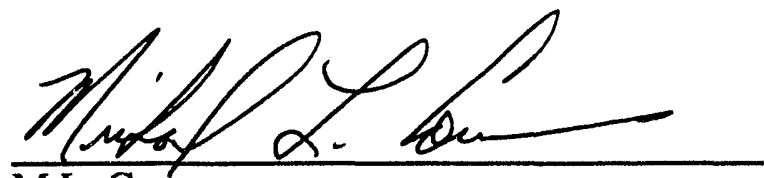

M.L. Cowen

Manager, Process Safety Technology

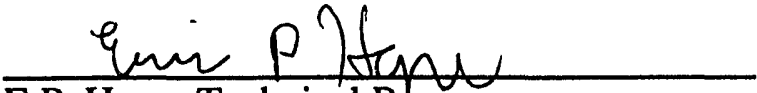

E.P. Hope, Technical R\&viewer

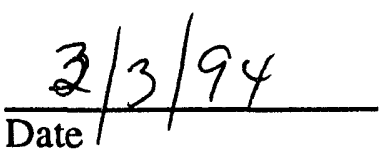

$\frac{3-3 \cdot 94}{\text { Date }}$
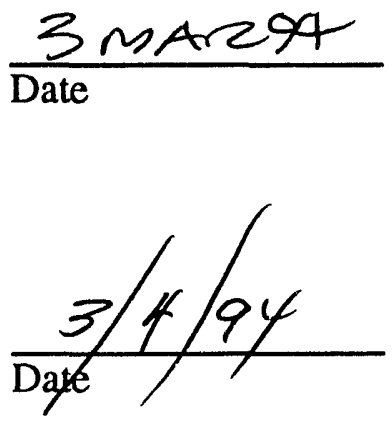

$\frac{7 \text { MAR } 94}{\text { Date }}$ 


\section{Introduction}

The Safety Analysis Report (SAR) Rev. 1 and Justification for Continued Operation (JCO) for HB-Line were written for the present mission of HB-Line to produce $\mathrm{Pu}-238$ oxide in support of the NASA Cassini mission 1,2. Both the SAR and the JCO were approved by DOE in 1992 prior to restart of the HB-Line Facility. An Environmental Assessment (EA) is currently being prepared for HB-Line. The EA reports consequence and risk information from the SAR and JCO. The HB-Line EA summarizes the doses for the following accidents; earthquake (ground level release) and propagated fire as in the JCO, and medium energetic and low energetic event as in the SAR. Four receptors are reported in the EA; maximum offsite individual, onsite worker, offsite population, and onsite population.

Additional analysis and/or an update of the present analysis is needed for the EA. The purpose of this report is to provide this necessary analysis. All of the changes result in a decrease to the doses as reported in the HB-Line SAR and JCO. Therefore, the SAR and JCO will not be updated at this time to incorporate these updated doses.

\section{Discussion}

The following is a summary of the updates that are reported in this report:

- Population Doses - Since the JCO and SAR do not include population doses for two accidents; a propagated fire and a ground level release for an earthquake, this analysis will provide these doses. In addition, the doses for medium energetic event and low energetic event will be updated to the new onsite and offsite population databases that have recently been incorporated into the AXAIR89Q code. The offsite population database used in the HB-Line SAR is based on the $1980 \mathrm{U}$. S. Census while the new database is based on the 1990 U. S. Census. For this analysis, the 1990 population distribution is projected to 1994 based on assumed growth rates for the area. The onsite population database that was used in the HB-Line SAR and JCO is based on a typical 1989 site population distribution while the new database is based on a typical 1992 site population distribution.

- Onsite Worker Dose (Co-located worker) - The SAR does not report doses to the onsite worker. The JCO analyzes the onsite worker at a distance of 100 meters from the release point using $99.5 \%$ meteorology for propagated fire and earthquake. This analysis will analyze the onsite worker at a distance of 640 meters from the release point using $50 \%$ meteorology in accordance with more recent DOE guidance. The doses will be calculated for all the accidents reported in the JCO.

- Maximum Offsite Individual Dose - Since the time the SAR and JCO were written, a new meteorological database has been incorporated into the AXAIR89Q dose code. The HB-Line SAR and JCO doses are calculated using the "old" meteorological database based on data collected during the five period of 1982 1986. The new meteorological database is based on data collected during the five year period of 1987 - 1991. This analysis will update the doses using the new meteorological database for all the accidents reported in the JCO. The new meteorological database results in a decrease in the dose from a release from $\mathrm{H}$-Area. 


\section{Analysis Method}

This analysis is not intended to reevaluate the source terms in the HB-Line SAR or JCO. A new SAR is currently being prepared in accordance with DOE Order 5480.23. New source terms will be addressed in the new SAR. This analysis only updates the consequences to the new meteorological and population databases using the same source terms as those in the HB-Line SAR and JCO.

The following assumptions are used in the analysis:

- The curies released to the environment are the same as those in the HB-Line SAR and JCO.

- The following worst-case curie fraction was used in the analysis. This is the same curie fraction used in the HB-Line SAR and JCO.

$\begin{array}{lc}\mathrm{Pu}-238 & \text { Curie Fraction } \\ \mathrm{Pu}-241 & 0.642 \\ 0.358\end{array}$

- The above curie fraction was used as the input to the AXAIR89Q consequence computer code, Version 1.2, for both a ground level and stack release from H-Area. Appendix A contains the AXAIR89Q input and output. The doses obtained from AXAIR89Q represent clearance class $W$, plutonium nitrate. The doses calculated for the Plutonium Oxide Facility use clearance class Y, plutonium oxide. To convert the AXAIR89Q doses from clearance class $W$ to $Y$ the doses from AXAIR89Q were multiplied by the ratio of dose factor for class $\mathrm{Y}$ to dose factor for class $\mathrm{W}$. The dose factors are from DOE/EH/--0071, Internal Dose Conversion Factors for Calculation of Dose to the Public ${ }^{3}$. The ratios are as follows:

$$
\begin{array}{ll}
\mathrm{Pu}-238 & \mathrm{DFY} / \mathrm{DFW}=300 / 460=0.652 \\
\mathrm{Pu}-239 & \mathrm{DFY} / \mathrm{DFW}=5.7 / 10=0.57
\end{array}
$$

Tables $1 \& 2$ summarize the doses per 1 curie release. Table 1 is used for the Scrap Recovery Facility doses, based on lung clearance class W (plutonium nitrate) and Table 2 is used for the Plutonium Oxide Facility doses, based on lung clearance class $\mathrm{Y}$ (plutonium oxide).

- The earthquake assumes a ground level release and all other accidents assume a release from the 61 meter stack.

\section{Results and Conclusion}

Table 3 summarizes the updated doses for earthquake. propagated fire, medium energetic event and low energetic event for inclusion in the HB-Line Environmental Assessment.

Tables $4 \& 5$ summarize the updated doses for all the accidents reported in the HB-Line JCO. Table 4 contains doses for the Scrap Recovery Facility and Table 5 contains doses for the Plutonium Oxide Facility.

Tables $6 \& 7$ summarize the updated maximum offsite individual risks for all the accidents reported in the HB-Line JCO. Table 6 contains risks for the Scrap Recovery Facility and Table 7 contains risks for the Plutonium Oxide Facility. 
In summary, this analysis updates the doses in the HB-Line JCO incorporating the following changes:

- Use of the new meteorological database in the AXAIR89Q consequence code based on the five year period of $1987-1991$.

- Use of the new offsite population database in the AXAIR89Q consequence code based on the 1990 U. S. Census.

- Use of the new onsite population database in the AXAIR89Q consequence code based on a typical 1992 site population distribution.

- Define the onsite worker (co-located worker) to be located 640 meters from the release point. Use 50\% meteorology for this receptor.

The net result of the above changes is a decrease in the total dose for all accidents analyzed in the SAR and JCO.

\section{References}

1. Safety Analysis - 200 Area. Separations Area Operations, Building 221-H B-Line. Scrap Recovery Facility and Plutonium Oxide Facility, Rev 1, DPSTSA-200-10-2, July 1991

2. Justification for Continued Operation. HB-Line, WSRC-RP-92-981, Westinghouse Savannah River Company, October 1992

3. Internal Dose Conversion Factors for Calculation of Dose to the Public, DOE/EH0071, U.S. Department of Energy, July 1988 
WSRC-RP-94-250

Table 1. Dose per 1 Curie Total Release from HB-Line (Lung Clearance Class W - Plutonium Nitrate)

\begin{tabular}{|c|c|c|c|c|c|}
\hline & \multirow[b]{2}{*}{$\begin{array}{l}\text { Curie } \\
\text { Fraction }\end{array}$} & \multicolumn{4}{|c|}{ Effective Dose Equivalent* } \\
\hline & & $\begin{array}{c}\text { MOI } \\
99.5 \% \mathrm{Met} \\
\text { (mrem }\end{array}$ & $\begin{array}{c}640 \mathrm{~m} \\
50 \% \mathrm{Met} \\
\mathrm{Ci})\end{array}$ & $\frac{\text { Offsite Pop }}{\text { (person }}$ & $\frac{\text { Onsite Pop }}{\text { rem/Ci }}$ \\
\hline Ground Level Release & & & & & \\
\hline Pu-238 & $6.42 E-01$ & $6.15 E+02$ & $4.96 E+03$ & $5.13 E+03$ & $3.08 E+03$ \\
\hline $\mathrm{Pu}-241$ & $3.58 \mathrm{E}-01$ & $7.45 E+00$ & $6.01 E+01$ & $6.22 \mathrm{E}+01$ & $3.73 E+01$ \\
\hline$\overline{\text { Total }}$ & $1.00 \mathrm{E}+00$ & $6.22 \mathrm{E}+02$ & $5.02 E+03$ & $5.19 E+03$ & $3.11 \mathrm{E}+03$ \\
\hline Stack Release & & & & & \\
\hline$\overline{\mathrm{Pu}-238}$ & $6.42 \mathrm{E}-01$ & $3.35 \mathrm{E}+02$ & $6.20 E+02$ & $4.06 \mathrm{E}+03$ & $1.40 E+03$ \\
\hline Pll-241 & $3.58 \mathrm{E}-01$ & $4.06 \mathrm{E}+00$ & $7.52 E+00$ & $4.92 \mathrm{E}+01$ & $1.70 \mathrm{E}+01$ \\
\hline$\overline{\text { Total }}$ & $1.00 \mathrm{E}+00$ & $3.39 \mathrm{E}+02$ & $6.28 E+02$ & $4.11 \mathrm{E}+03$ & $1.42 \mathrm{E}+03$ \\
\hline
\end{tabular}

MOI - Maximum Offsite Individual

Table 2. Dose per 1 Curie Total Release from HB-Line (Lung Clearance Class Y - Plutonium Oxide)

\begin{tabular}{|c|c|c|c|c|c|}
\hline & \multirow{3}{*}{$\begin{array}{l}\text { Curie } \\
\text { Fraction }\end{array}$} & \multicolumn{4}{|c|}{ Effective Dose Equivalent* } \\
\hline & & $\begin{array}{c}\text { MOI } \\
99.5 \% \mathrm{Met}\end{array}$ & $\begin{array}{c}640 \mathrm{~m} \\
50 \% \mathrm{Met}\end{array}$ & Offsite Pop & Onsite Pop \\
\hline & & \multicolumn{2}{|c|}{ (mrem/Ci) } & \multicolumn{2}{|c|}{ (person rem/Ci) } \\
\hline Ground Level Release & & & & & \\
\hline$\overline{\mathrm{Pu}-238}$ & $6.42 \mathrm{E}-01$ & $4.01 E+02$ & $3.23 E+03$ & $3.35 E+03$ & $2.01 E+03$ \\
\hline $\mathrm{Pu}-241$ & $3.58 \mathrm{E}-01$ & $4.25 E+00$ & $3.43 E+01$ & $3.55 E+01$ & $2.13 E+01$ \\
\hline Total & $1.00 \mathrm{E}+00$ & $4.05 \mathrm{E}+02$ & $3.27 \mathrm{E}+03$ & $3.38 \mathrm{E}+03$ & $2.03 E+03$ \\
\hline \multicolumn{6}{|l|}{ Stack Release } \\
\hline$\overline{P u-238}$ & $6.42 \mathrm{E}-01$ & $2.18 E+02$ & $4.04 \mathrm{E}+02$ & $2.65 \mathrm{E}+03$ & $9.13 E+02$ \\
\hline $\mathrm{Pu}-241$ & $3.58 \mathrm{E}-01$ & $2.31 E+00$ & $4.29 E+00$ & $2.80 \mathrm{E}+01$ & $9.69 E+00$ \\
\hline Total & $1.00 \mathrm{E}+00$ & $2.21 \mathrm{E}+02$ & $4.09 \mathrm{E}+02$ & $2.68 E+03$ & $9.23 \mathrm{E}+02$ \\
\hline
\end{tabular}

MOI - Maximum Offsite Individual

*AXAIR89Q Version 1.2 - 1987 - 1991 meteorological database

Offsite population based on 1990 U.S. Census

Onsite population based on 1992 population database 
Table 3. Summary of Consequences for the HB-Line Environmental Assessment

Scrap Recovery Facility

\begin{tabular}{lccccc}
\hline \hline & & \multicolumn{3}{c}{ Effective Dose Equivalent } \\
\cline { 3 - 6 } & MoI & $640 \mathrm{~m}$ & \\
& Curie & $99.5 \% \mathrm{Met}$ & $50 \%$ Met & Offsite Pop Onsite Pop \\
\cline { 3 - 6 } & Release & \multicolumn{2}{c}{ (mrem) } & \multicolumn{2}{c}{ (person rem) } \\
\hline Earthquake & $1.49 \mathrm{E}-02$ & $9.27 \mathrm{E}+00$ & $7.48 \mathrm{E}+01$ & $7.74 \mathrm{E}+01$ & $4.63 \mathrm{E}+01$ \\
Propagated Fire & $2.95 \mathrm{E}-01$ & $1.00 \mathrm{E}+02$ & $1.85 \mathrm{E}+02$ & $1.21 \mathrm{E}+03$ & $4.18 \mathrm{E}+02$ \\
Medium Energetic Event & $3.47 \mathrm{E}-02$ & $1.18 \mathrm{E}+01$ & $2.18 \mathrm{E}+01$ & $1.43 \mathrm{E}+02$ & $4.92 \mathrm{E}+01$ \\
Low Energetic Event & $1.73 \mathrm{E}-02$ & $5.87 \mathrm{E}+00$ & $1.09 \mathrm{E}+01$ & $7.11 \mathrm{E}+01$ & $2.45 \mathrm{E}+01$ \\
\hline
\end{tabular}

Plutonium Oxide Facility

\begin{tabular}{lccccc}
\hline \hline & & \multicolumn{4}{c}{ Effective Dose Equivalent } \\
\cline { 3 - 6 } & Curie & MOI & $640 \mathrm{~m}$ & \\
\cline { 3 - 6 } & Release & \multicolumn{2}{c}{ (mrem) } & $50 \%$ Met & Offsite Pop Onsite Pop \\
\cline { 3 - 6 } & $6.13 \mathrm{E}-03$ & $2.48 \mathrm{E}+00$ & $2.00 \mathrm{E}+01$ & \multicolumn{2}{c}{ (person rem) } \\
\hline Earthquake & $1.21 \mathrm{E}-01$ & $2.67 \mathrm{E}+01$ & $4.94 \mathrm{E}+01$ & $3.24 \mathrm{E}+02$ & $1.24 \mathrm{E}+01$ \\
Propagated Fire & $1.92 \mathrm{E}-02$ & $4.24 \mathrm{E}+00$ & $7.85 \mathrm{E}+00$ & $5.14 \mathrm{E}+01$ & $1.77 \mathrm{E}+01$ \\
Medium Energetic Event & $1.53 \mathrm{E}-04$ & $2.10 \mathrm{E}-01$ & $3.89 \mathrm{E}-01$ & $2.55 \mathrm{E}+00$ & $8.79 \mathrm{E}-01$ \\
Low Energetic Event & $9.53 \mathrm{E}-04$ & & & &
\end{tabular}

MOI - Maximum Offsite Individual 
Table 4. Scrap Recovery Facility - Consequences Updated to New Meteorological and Population Databases AXAIR89Q, Version 1.2

\begin{tabular}{lccccc}
\hline \hline & & \multicolumn{4}{c}{ Effective Dose Equivalent } \\
\cline { 3 - 7 } & Curie & $99.5 \%$ Met & $50 \%$ Met & Offsite Pop & Onsite Pop \\
\cline { 3 - 7 } & Release & \multicolumn{2}{c}{ (mrem) } & \multicolumn{2}{c}{ (person rem) } \\
\cline { 3 - 7 } & $1.73 E-02$ & $5.87 \mathrm{E}+00$ & $1.09 \mathrm{E}+01$ & $7.11 \mathrm{E}+01$ & $2.45 \mathrm{E}+01$ \\
& $1.73 \mathrm{E}-04$ & $5.87 \mathrm{E}-02$ & $1.09 \mathrm{E}-01$ & $7.11 \mathrm{E}-01$ & $2.45 \mathrm{E}-01$ \\
LE (both HEPA stages fail) & $1.73 \mathrm{E}-04$ & $5.87 \mathrm{E}-02$ & $1.09 \mathrm{E}-01$ & $7.11 \mathrm{E}-01$ & $2.45 \mathrm{E}-01$ \\
LE (stage 1 HEPA fails) & $3.47 \mathrm{E}-02$ & $1.18 \mathrm{E}+01$ & $2.18 \mathrm{E}+01$ & $1.43 \mathrm{E}+02$ & $4.92 \mathrm{E}+01$ \\
LE (stage 2 HEPA fails) & $1.85 \mathrm{E}-04$ & $6.27 \mathrm{E}-02$ & $1.16 \mathrm{E}-01$ & $7.60 \mathrm{E}-01$ & $2.62 \mathrm{E}-01$ \\
ME (both HEPA stages fail) & $7.35 \mathrm{E}-08$ & $2.49 \mathrm{E}-05$ & $4.61 \mathrm{E}-05$ & $3.02 \mathrm{E}-04$ & $1.04 \mathrm{E}-04$ \\
ME (room B') & $1.73 \mathrm{E}-06$ & $5.87 \mathrm{E}-04$ & $1.09 \mathrm{E}-03$ & $7.11 \mathrm{E}-03$ & $2.45 \mathrm{E}-03$ \\
RAR (room B') & $3.47 \mathrm{E}-04$ & $1.18 \mathrm{E}-01$ & $2.18 \mathrm{E}-01$ & $1.43 \mathrm{E}+00$ & $4.92 \mathrm{E}-01$ \\
LE (cabinet B) & $3.47 \mathrm{E}-04$ & $1.18 \mathrm{E}-01$ & $2.18 \mathrm{E}-01$ & $1.43 \mathrm{E}+00$ & $4.92 \mathrm{E}-01$ \\
ME (stage 1 HEPA fails) & $6.40 \mathrm{E}-12$ & $2.17 \mathrm{E}-09$ & $4.02 \mathrm{E}-09$ & $2.63 \mathrm{E}-08$ & $9.07 \mathrm{E}-09$ \\
ME (stage 2 HEPA fails) & $1.49 \mathrm{E}-02$ & $9.27 \mathrm{E}+00$ & $7.48 \mathrm{E}+01$ & $7.74 \mathrm{E}+01$ & $4.63 \mathrm{E}+01$ \\
RAR (cabinet B) & $3.47 \mathrm{E}-06$ & $1.18 \mathrm{E}-03$ & $2.18 \mathrm{E}-03$ & $1.43 \mathrm{E}-02$ & $4.92 \mathrm{E}-03$ \\
Earthquake (grd release) & $6.40 \mathrm{E}-08$ & $2.17 \mathrm{E}-05$ & $4.02 \mathrm{E}-05$ & $2.63 \mathrm{E}-04$ & $9.07 \mathrm{E}-05$ \\
ME (cabinet B) & $6.40 \mathrm{E}-10$ & $2.17 \mathrm{E}-07$ & $4.02 \mathrm{E}-07$ & $2.63 \mathrm{E}-06$ & $9.07 \mathrm{E}-07$ \\
RAR (both HEPA stages fail) & $6.40 \mathrm{E}-10$ & $2.17 \mathrm{E}-07$ & $4.02 \mathrm{E}-07$ & $2.63 \mathrm{E}-06$ & $9.07 \mathrm{E}-07$ \\
RAR (stage 1 HEPA fails) & & & & &
\end{tabular}

LE - Low Energetic Event

ME - Medium Energetic Event

RAR - Residual Release Activity 
Table 5. Plutonium Oxide Facility - Consequences Updated to New Meteorological and Population Databases AXAIR89Q, Version 1.2

\begin{tabular}{|c|c|c|c|c|c|}
\hline & \multirow{3}{*}{$\begin{array}{c}\text { Curie } \\
\text { Release }\end{array}$} & \multicolumn{4}{|c|}{ Effective Dose Equivalent } \\
\hline & & $\begin{array}{c}\text { MOI } \\
99.5 \% \mathrm{Met}\end{array}$ & $\begin{array}{c}640 \mathrm{~m} \\
50 \% \mathrm{Met}\end{array}$ & Offsite Pop & Onsite Pop \\
\hline & & \multicolumn{2}{|c|}{ (mrem) } & \multicolumn{2}{|c|}{ (person rem) } \\
\hline LE (both HEPA stages fail) & $9.53 \mathrm{E}-04$ & $2.10 \mathrm{E}-01$ & $3.89 \mathrm{E}-01$ & $2.55 \mathrm{E}+00$ & $8.79 \mathrm{E}-01$ \\
\hline ME (both HEPA stages fail) & $1.92 \mathrm{E}-02$ & $4.24 E+00$ & $7.85 E+00$ & $5.14 E+01$ & $1.77 E+01$ \\
\hline AL (both HEPA stages fail) & $7.38 \mathrm{E}-04$ & 1.63E-01 & $3.02 \mathrm{E}-01$ & $1.97 \mathrm{E}+00$ & 6.81E-01 \\
\hline RAR (room B') & 4.95E-08 & 1.09E-05 & $2.02 \mathrm{E}-05$ & $1.32 \mathrm{E}-04$ & 4.57E-05 \\
\hline $\mathrm{ME}$ (room B') & $9.90 \mathrm{E}-05$ & $2.19 \mathrm{E}-02$ & 4.05E-02 & $2.65 \mathrm{E}-01$ & $9.14 \mathrm{E}-02$ \\
\hline LE (stage 1 HEPA fails) & 9.53E-06 & $2.10 \mathrm{E}-03$ & $3.89 \mathrm{E}-03$ & $2.55 \mathrm{E}-02$ & 8.79E-03 \\
\hline LE (stage 2 HEPA fails) & $9.53 E-06$ & $2.10 \mathrm{E}-03$ & $3.89 \mathrm{E}-03$ & 2.55E-02 & 8.79E-03 \\
\hline ME (stage 1 HEPA fails) & $1.92 \mathrm{E}-04$ & 4.24E-02 & $7.85 \mathrm{E}-02$ & $5.14 \mathrm{E}-01$ & $1.77 \mathrm{E}-01$ \\
\hline ME (stage 2 HEPA fails) & $1.92 \mathrm{E}-04$ & 4.24E-02 & $7.85 \mathrm{E}-02$ & $5.14 \mathrm{E}-01$ & $1.77 \mathrm{E}-01$ \\
\hline Earthquake (grd release) & $6.13 E-03$ & $2.48 E+00$ & $2.00 \mathrm{E}+01$ & $2.07 \mathrm{E}+01$ & $1.24 E+01$ \\
\hline LE (cabinet B) & 9.53E-08 & 2.10E-05 & $3.89 \mathrm{E}-05$ & 2.55E-04 & 8.79E-05 \\
\hline ME (cabinet B) & $1.92 \mathrm{E}-06$ & 4.24E-04 & $7.85 \mathrm{E}-04$ & $5.14 \mathrm{E}-03$ & $1.77 \mathrm{E}-03$ \\
\hline RAR (both HEPA stages fail) & $4.58 \mathrm{E}-08$ & $1.01 E-05$ & $1.87 \mathrm{E}-05$ & $1.23 \mathrm{E}-04$ & 4.23E-05 \\
\hline RAR (cabinet B) & $4.58 \mathrm{E}-12$ & 1.01E-09 & $1.87 \mathrm{E}-09$ & $1.23 E-08$ & 4.23E-09 \\
\hline RAR (stage 1 HEPA fails) & $4.58 \mathrm{E}-10$ & 1.01E-07 & 1.87E-07 & $1.23 \mathrm{E}-06$ & 4.23E-07 \\
\hline RAR (stage 2 HEPA fails) & $4.58 E-10$ & 1.01E-07 & $1.87 E-07$ & $1.23 \mathrm{E}-06$ & 4.23E-07 \\
\hline
\end{tabular}

LE - Low Energetic Event

ME - Medium Energetic Event

RAR - Residual Release Activity 
Table 6 Scrap Recovery Facility - Maximum Offsite Individual Risk Updated to New Meteorological and Population Databases AXAIR89Q, Version 1.2

\begin{tabular}{lccc}
\hline \hline & $\begin{array}{c}\text { MOI Dose } \\
\text { (mrem) }\end{array}$ & $\begin{array}{c}\text { Frequency* } \\
\text { (per year) }\end{array}$ & $\begin{array}{c}\text { Risk } \\
\text { (mrem/yr) }\end{array}$ \\
\hline LE (both HEPA stages fail) & $5.87 \mathrm{E}+00$ & $2.10 \mathrm{E}-01$ & $1.23 \mathrm{E}+00$ \\
LE (stage 1 HEPA fails) & $5.87 \mathrm{E}-02$ & $2.20 \mathrm{E}+00$ & $1.29 \mathrm{E}-01$ \\
LE (stage 2 HEPA fails) & $5.87 \mathrm{E}-02$ & $2.20 \mathrm{E}+00$ & $1.29 \mathrm{E}-01$ \\
ME (both HEPA stages fail) & $1.18 \mathrm{E}+01$ & $3.70 \mathrm{E}-03$ & $4.35 \mathrm{E}-02$ \\
ME (room B') & $6.27 \mathrm{E}-02$ & $4.30 \mathrm{E}-01$ & $2.70 \mathrm{E}-02$ \\
RAR (room B') & $2.49 \mathrm{E}-05$ & $1.30 \mathrm{E}+02$ & $3.24 \mathrm{E}-03$ \\
LE (cabinet B) & $5.87 \mathrm{E}-04$ & $2.40 \mathrm{E}+01$ & $1.41 \mathrm{E}-02$ \\
ME (stage 1 HEPA fails) & $1.18 \mathrm{E}-01$ & $4.00 \mathrm{E}-02$ & $4.71 \mathrm{E}-03$ \\
ME (stage 2 HEPA fails) & $1.18 \mathrm{E}-01$ & $4.00 \mathrm{E}-02$ & $4.71 \mathrm{E}-03$ \\
RAR (cabinet B) & $2.17 \mathrm{E}-09$ & $1.30 \mathrm{E}+02$ & $2.82 \mathrm{E}-07$ \\
Earthquake (grd release) & $9.27 \mathrm{E}+00$ & $1.70 \mathrm{E}-04$ & $1.58 \mathrm{E}-03$ \\
ME (cabinet B) & $1.18 \mathrm{E}-03$ & $4.30 \mathrm{E}-01$ & $5.06 \mathrm{E}-04$ \\
RAR (both HEPA stages fail) & $2.17 \mathrm{E}-05$ & $1.10 \mathrm{E}+00$ & $2.39 \mathrm{E}-05$ \\
RAR (stage 1 HEPA fails) & $2.17 \mathrm{E}-07$ & $1.20 \mathrm{E}+01$ & $2.60 \mathrm{E}-06$ \\
RAR (stage 2 HEPA fails) & $2.17 \mathrm{E}-07$ & $1.20 \mathrm{E}+01$ & $2.60 \mathrm{E}-06$ \\
\hline
\end{tabular}

* Frequency from SAR

LE - Low Energetic Event

ME - Medium Energetic Event

RAR - Residual Release Activity 
Table 7 Plutonium Oxide Facility - Maximum Offsite Individual Risk Updated to New Meteorological and Population Databases AXAIR89Q, Version 1.2

\begin{tabular}{lccc}
\hline \hline & $\begin{array}{c}\text { MOI Dose } \\
\text { (mrem) }\end{array}$ & $\begin{array}{c}\text { Frequency* } \\
\text { (per year) }\end{array}$ & $\begin{array}{c}\text { Risk } \\
\text { (mrem/yr) }\end{array}$ \\
\hline LE (both HEPA stages fail) & $2.10 \mathrm{E}-01$ & $6.00 \mathrm{E}-02$ & $1.26 \mathrm{E}-02$ \\
ME (both HEPA stages fail) & $4.24 \mathrm{E}+00$ & $7.00 \mathrm{E}-04$ & $2.97 \mathrm{E}-03$ \\
AL (both HEPA stages fail) & $1.63 \mathrm{E}-01$ & $1.40 \mathrm{E}-02$ & $2.28 \mathrm{E}-03$ \\
RAR (room B') & $1.09 \mathrm{E}-05$ & $1.70 \mathrm{E}+02$ & $1.86 \mathrm{E}-03$ \\
ME (room B') & $2.19 \mathrm{E}-02$ & $8.00 \mathrm{E}-02$ & $1.75 \mathrm{E}-03$ \\
LE (stage 1 HEPA fails) & $2.10 \mathrm{E}-03$ & $6.00 \mathrm{E}-01$ & $1.26 \mathrm{E}-03$ \\
LE (stage 2 HEPA fails) & $2.10 \mathrm{E}-03$ & $6.00 \mathrm{E}-01$ & $1.26 \mathrm{E}-03$ \\
ME (stage 1 HEPA fails) & $4.24 \mathrm{E}-02$ & $7.00 \mathrm{E}-03$ & $2.97 \mathrm{E}-04$ \\
ME (stage 2 HEPA fails) & $4.24 \mathrm{E}-02$ & $7.00 \mathrm{E}-03$ & $2.97 \mathrm{E}-04$ \\
Earthquake (grd release) & $2.48 \mathrm{E}+00$ & $1.70 \mathrm{E}-04$ & $4.22 \mathrm{E}-04$ \\
LE (cabinet B) & $2.10 \mathrm{E}-05$ & $6.00 \mathrm{E}+00$ & $1.26 \mathrm{E}-04$ \\
ME (cabinet B) & $4.24 \mathrm{E}-04$ & $7.00 \mathrm{E}-02$ & $2.97 \mathrm{E}-05$ \\
RAR (both HEPA stages fail) & $1.01 \mathrm{E}-05$ & $1.00 \mathrm{E}+00$ & $1.01 \mathrm{E}-05$ \\
RAR (cabinet B) & $1.01 \mathrm{E}-09$ & $1.40 \mathrm{E}+02$ & $1.42 \mathrm{E}-07$ \\
RAR (stage 1 HEPA fails) & $1.01 \mathrm{E}-07$ & $1.00 \mathrm{E}+01$ & $1.01 \mathrm{E}-06$ \\
RAR (stage 2 HEPA fails) & $1.01 \mathrm{E}-07$ & $1.00 \mathrm{E}+01$ & $1.01 \mathrm{E}-06$ \\
\hline \hline
\end{tabular}

*Frequency from SAR

LE - Low Energetic Event

ME - Medium Energetic Event

RAR - Residual Release Activity 
Appendix A

AXAIR89Q

Input \& Output

A-1 


\section{c1:4:74}

$* * * * * * * * * * * * * * * * * * * * * * * * * * * * * * * * * * * * * * * * * * * * * * *$
$* *$
AXAIR VERSION 1.2
$*$
$*$
SRTC DOSE ASSESSMENT OF A POSTULATED

THE LOCATION OF THE RELEASE IS H AREA (AT ASSUMED SRS-GRID COORDINATES: EAST 64257. FT; NORTH 70415. FT;. THE FELEASE IS FROM A STACK HEIGHT OF 61. METERS ABOVE GRADE (GRADE ELEVATION SAME AS ADJACENT TERRAंN.) THE CALENDAR YEAR FOR THE POSTULATED RELEASE IS 1994

i THE AIXLLYSIS IS FOR ALL 16 COMPASS SECTORS.

DAUEHTER INISIOWTH WILL BE LIMITED TO PARENTS WITH A HALF-LIFE LESS THAN 24 HOURS.

50\% INOIVIDUAL AND 99.5\% INDIVIDUAL \& POPULATINN DOSE CALCULATIONS WILL BE PERFORMED ICAF: DOSE VALUES WERE USED IN THE CALCULATIONS. USE ONLY ADULT DOSES WITH ICRP3O. $1987-1991$ METEOROLOGY USED IN THE CALCULATIONS.

OUE T: MAXIMUM INDIVIDUAL DOSES ARE GIVEN AT 1 DISTANCES FROM THE SOURCE.

DISTANCES FFIOM THE SOURCE, IN MILES, ARE:

GAMMA-SHINE METHOD: NONUNIFORM PLUME UPPER-BOUND AFPROXIMATION.

NUMBER OF RELEASED RADIONUCLIDES $=2$

TABLE OF RELEASES OF RADIOACTIVITY

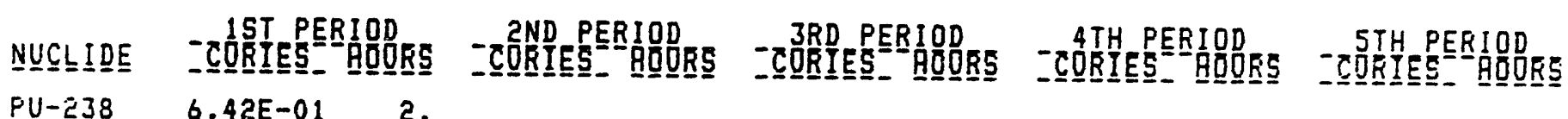

$\begin{array}{lll}P U-238 & 6.42 E-01 & 2 . \\ P U-241 & 3.58 E-01 & 2\end{array}$ 


\section{Stack Releas E \\ $2 / 25 / 94$ \\ $11: 39$}

SUMMARY OF WORST-SECTOR, WORST-CASE (99.5\%) DOSE VALUES (REM OR PERSON-REM)

\begin{tabular}{|c|c|c|c|c|c|}
\hline & EFF.D.E. & ORG. DOSE & ORGAN & AGE/DIST & SECTOR \\
\hline OFFSITE MAXIMUM INDIVIDUAL & $3.39 E-01$ & $5.97 E+00$ & B. SURF. & ADULT & NW \\
\hline OFFSITE POPULATION (50 MILES) & $4.11 E+03$ & $7.24 E+04$ & B. SURF. & & WNW \\
\hline ONSITE POPULATION & $1.42 E+03$ & $2.50 E+04$ & B. SURF. & & WNW \\
\hline $\begin{array}{l}\text { AREA (I MILE RADIUS) MAX IND } \\
\text { (USING GIVEN DIST. C I MILEF }\end{array}$ & $2.46 E+00$ & $4.34 E+01$ & B. SURF. & $\begin{array}{l}\text { M. } \\
\text { MILES }\end{array}$ & WSW \\
\hline AREA (I MILE) POPULATION DOSE & $9.66 E+02$ & $1.70 \mathrm{E}+04$ & B. SURF. & & WNW \\
\hline
\end{tabular}

SUMMARY OF 50\% PROBABILITY DOSE VALUES (REM OR PERSON-REM)

\begin{tabular}{l} 
OFFSITE MAXIMUM INDIUIDUAL $6.59 E-02$ \\
\hline AREA (I MILE RADIUS) MAX IND. \\
ISAME DISTANCE AS WORST DOSE)
\end{tabular}




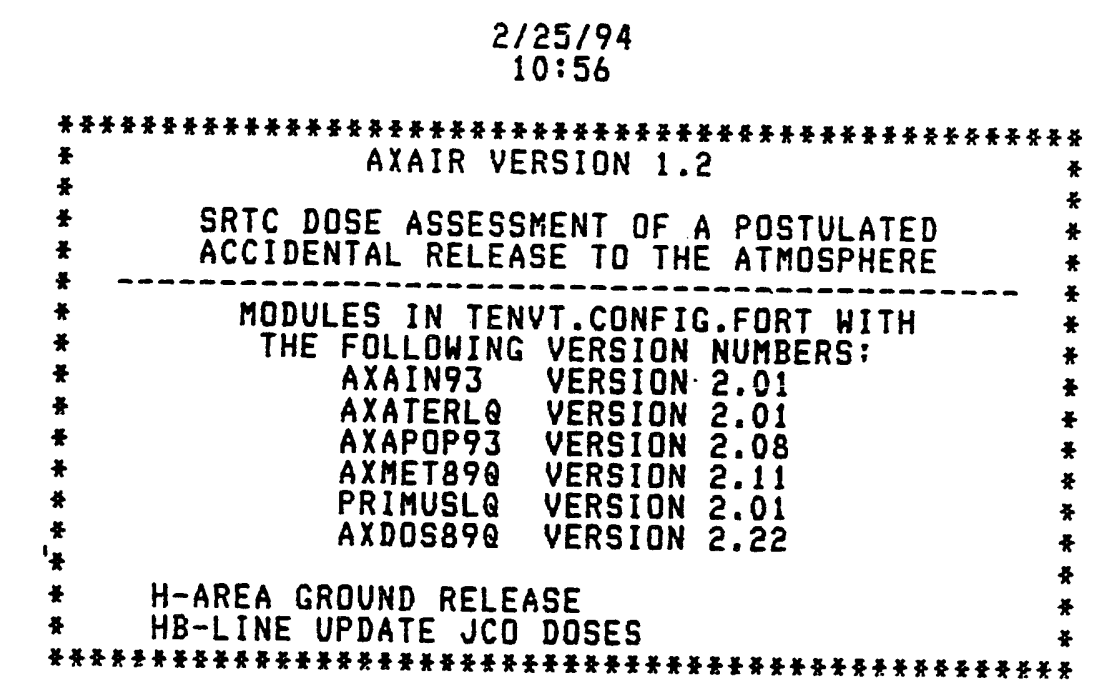

THE LOCATION OF THE RELEASE IS H AREA (AT ASSUMED SRS-GRID COORDINATES: EAST 64257. FT: NORTH 704:5. FT). THE RELEASE IS FROM GROUND LEVEL OR A BUILDING VENT (BLDG MINIMUM VERTICAL $X-S E C T I O N=$

P THE CALENDAR YEAR FOR THE POSTULATED RELEASE IS 1994

THE ANALYSIS IS FOR ALL 16 COMPASS SECTORS.

DAUGHTER INGROWTH WILL EE LIMITED TO FARENTS WITH A HALF-LIFE LESS THAN 24 HOURS.

50\% INUIVIDUAL AND 99.5\% INDIVIDUAL \& POPULATION DOSE CALCULATIONS WILL BE PERFORMED ICRP3O DOSE VALUES WERE USED IN THE CALCULATIONS. USE ONLY ADULT DOSES WITH ICRPJO. 1987-1991 METEOROLOGY USED IN THE CALCULATIONS.

ONSITE MAXIMUM INDIVIDUAL DOSES ARE GIVEN AT 1 DISTANCES FROM THE SOURCE.

DISTANCES FROM THE SOURCE, IN MILES, ARE:

GAMMA-SHINE METHOD: NONUNIFORM PLUME UPPER-BOUND APPROXIMATION.

NUMBER OF RELEASED RADIONUCLIDES $=2$

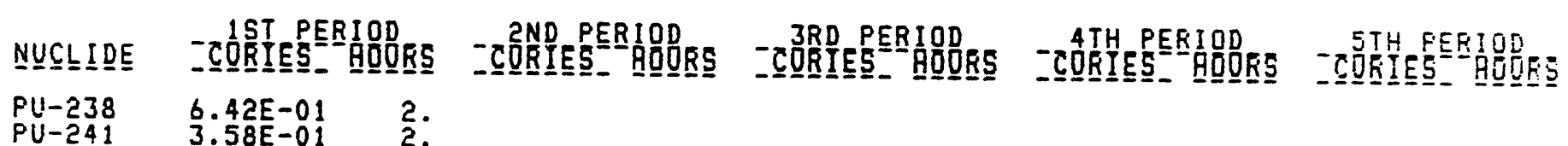




\section{GROUND RELEASE}

\section{$2 / 25 / 94$}

$11: 22$

SUMMARY OF WORST-SECTOR, WORST-CASE (99.5\%) DOSE VALUES (REM OR PERSON-REM)

\begin{tabular}{|c|c|c|c|c|c|}
\hline & EFF.D.E. & ORG. DOSE & ORGAN & AGE/DIST & SECTOR \\
\hline OFFSITE MAXIMUM INDIVIDUAL & $6.22 E-01$ & $1.10 E+01$ & B. SURF. & ADULT & $\mathrm{N}$ \\
\hline OFFSITE POPULATION (50 MILES) & $5.19 E+03$ & $9.15 E+04$ & B. SURF. & & WNW \\
\hline ONSITF POPULATION & $3.11 E+03$ & $5.49 E+04$ & B. SUFF. & & WNW \\
\hline $\begin{array}{l}\text { AREA (1 MILE TADIUSI MAX IND } \\
\text { OUSING GIVEN DIST. S I MILE) }\end{array}$ & $1.53 E+01$ & $2.70 E+02$ & B. SURF. & $\begin{array}{l}\text { MO } \\
\text { MILES }\end{array}$ & NE \\
\hline AREA (I MILE) POPULATION DOSE & $2.47 E+03$ & $4.36 E+04$ & B. SURF & & WNW \\
\hline
\end{tabular}

SUMMARY OF 50\% FROBABILITY DOSE VALUES (REM OR PERSON-REM).

\begin{tabular}{l} 
OFFSITE MAXIMUA INDIVIDUAL \\
AREA (1 MILE RADIUSI MAX IND \\
ISAME DISTANCE AS WORST DOSE) \\
\hline
\end{tabular}



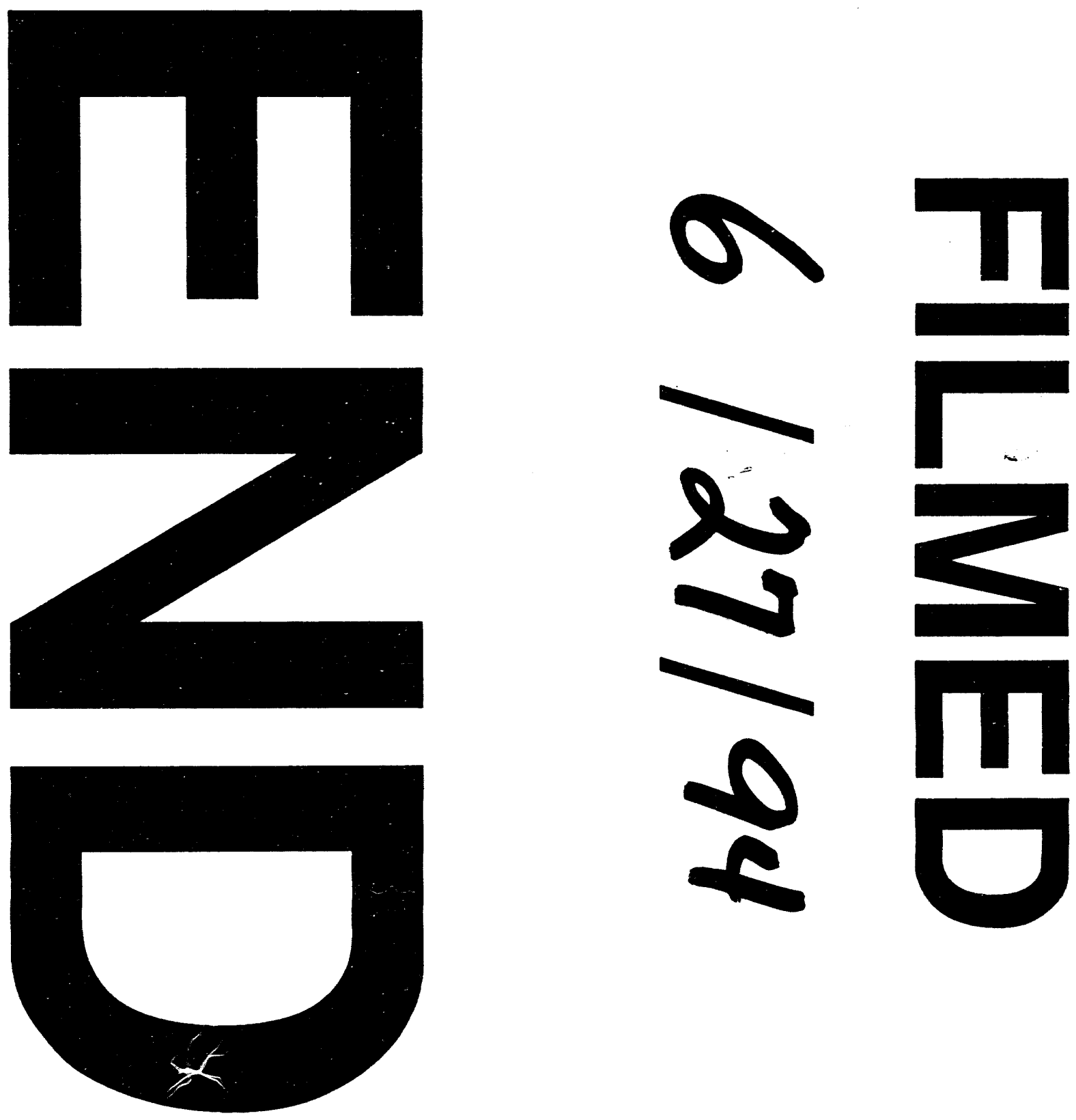
$\therefore 0$

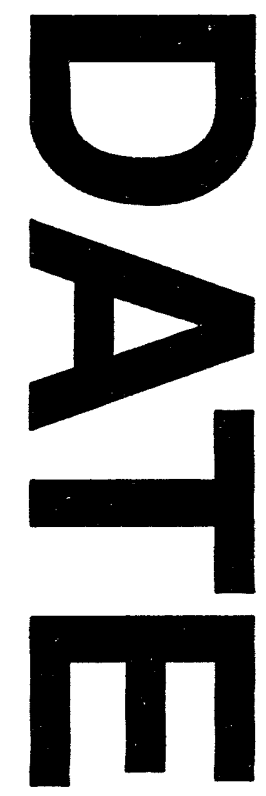


